\title{
BMJ Open A prospective cohort study characterising the role of anogenital warts in HIV acquisition among men who have sex with men: a study protocol
}

\author{
Brandon Brown, ${ }^{1}$ Mariam Davtyan, ${ }^{1}$ Segundo R Leon, ${ }^{2}$ Hugo Sanchez, ${ }^{3}$ \\ Gino Calvo, ${ }^{3}$ Jeffrey D Klausner, ${ }^{4}$ Jerome Galea ${ }^{3}$
}

To cite: Brown B, Davtyan M, Leon SR, et al. A prospective cohort study characterising the role of anogenital warts in HIV acquisition among men who have sex with men: a study protocol. BMJ Open 2014;4:e005687.

doi:10.1136/bmjopen-2014005687

- Prepublication history for this paper is available online To view these files please visit the journal online (http://dx.doi.org/10.1136/ bmjopen-2014-005687).

Received 12 May 2014 Revised 11 August 2014 Accepted 18 August 2014

CrossMark

For numbered affiliations see end of article.

Correspondence to Dr Brandon Brown; brandon.brown@uci.edu

\section{ABSTRACT}

Introduction: The HIV epidemic in Latin America is concentrated among men who have sex with men (MSM) and transgender women (TGW) with transmission predominately occurring during unprotected anal intercourse. This mode of transmission is also responsible for other sexually transmitted infections (STIs) such as herpes simplex, chlamydia and gonorrhoea, human papillomavirus (HPV)/genital warts and syphilis. Studies assessing the prevalence of HIV and HPV among MSM have not addressed the role of genital warts and HPV-related diseases in the acquisition of HIV infection. Community-based testing programmes are a potentially important way to remove barriers including stigma for individuals to learn about their STI status.

Methods and analysis: The prospective cohort study will recruit $600 \mathrm{MSM} / \mathrm{TGW}$ at a community centre in Lima, Peru, named Epicentro. Half of the participants will have a history of or have current anogenital warts (AGW), and the other half will have no history of AGW. We will measure the prevalence and acquisition of STIs including syphilis, HPV, chlamydia and gonorrhoea and the HIV-incidence in the two groups. To the best of our knowledge, it will be the first study that specifically examines the impact of genital warts on incident HIV infection. This study will help to understand the relationship between AGW and HIV infection among MSM/TGW in Peru. Furthermore, it may facilitate the development of preventive intervention strategies to reduce the prevalence of AGW and prevent incident HIV infection. HPV-related manifestations may be a good proxy for HIV risk.

Ethics and dissemination: This study was approved by institutional review boards at the University of California, Los Angeles (UCLA) in the USA and Impacta in Peru. Study findings will be shared with the Peruvian Ministry of Health as well as other international and national public health organisations. Study results will be translated into Spanish for participants.

Trial registration number: The Clinicaltrials.gov registration number is NCT01387412

\section{Strengths and limitations of this study}

Previous studies have examined sexually transmitted infection/HIV coinfection, but genital warts were not examined specifically for HIV risk.

- Results from this study may facilitate the development of proactive intervention strategies to reduce prevalence of anogenital warts and hence prevent incident HIV infection.

- Focuses on a population in high need (high HIV prevalence) of HIV prevention interventions in Peru.

- Visible genital warts were treated, so the risk of HIV in this group may have been decreased with the treatment.

- Loss to follow-up of participants is possible in this longitudinal study, and if there are few HIV cases over a 2-year period this may skew our results.

\section{INTRODUCTION}

Persons with HIV infection are at a higher risk of becoming infected with the human papillomavirus (HPV) compared with those who are HIV negative. The contrary is also true: individuals infected with HPV may be more likely to acquire HIV. This has been shown to be true among men who have sex with men (MSM) in a recent review. ${ }^{1}$ However, the role of the clinical manifestation of HPV-related anogenital warts (AGW) on HIV acquisition is currently unknown. ${ }^{2}$ Other sexually transmitted infections (STIs) have long been associated with HIV acquisition. ${ }^{3}$ For instance, syphilis ${ }^{4}$ chlamydia and trichomonas ${ }^{5}$ cancroid, ${ }^{6}$ gonorrhoea ${ }^{7}$ and herpes simplex virus 2 (HSV2) ${ }^{8}$ have all been shown to increase the likelihood of HIV infection. Few studies have shown that AGW are independently associated with HIV acquisition, ${ }^{9}$ fewer in men who have sex with men (MSM) and transgender women (TGW), populations heavily burdened by HPV-related disease. ${ }^{10}$

MSM/TGW are particularly vulnerable to rectal STIs due to the practice of receptive 
anal intercourse which has contributed to a high prevalence of HPV in these populations. In a recent systematic review and meta-analysis which provided estimates of the prevalence of anal canal HPV in HIV-uninfected and infected MSM, Machalek $e t a l^{11}$ presented a report indicating a high proportion of anal HPV among HIV-infected and uninfected MSM, 89-93\% and 54-64\% respectively. A study by Donà $e t a l^{12}$ reported that $74.8 \%$ of HIV-negative MSM had anal HPV illustrating a high prevalence of HPV among this high-risk population group. A longitudinal study of MSM conducted by Chin-Hong et al, ${ }^{13}$ and aimed at assessing the relationship between anal HPV and HIV acquisition, found that multiple HPV infections with at least two HPV types was associated with HIV acquisition with an adjusted hazard rate of 3.5 (95\% CI 1.2 to 10.6$)$.

In Latin America, the vast majority of HIV infections are among MSM/TGW, with transmission occurring during unprotected anal intercourse. ${ }^{14}{ }^{15}$ Specifically in South America, the prevalence of HIV infection in $\mathrm{MSM} / \mathrm{TGW}$ populations in most major cities is between $5 \%$ and $20 \%$, generally much higher than in the general adult population in which it is usually under $1 \% .{ }^{16}{ }^{17} \mathrm{In}$ Peru, the HIV prevalence in MSM ranges from 9.5 to $10.5 \%^{18}{ }^{19}$ with a prevalence of nearly $30 \%$ in TGW (REF MOH 2011, Silva-Santisteban Universidad Peruana Cayetano Heredia (UPCH)). At the Gay Men's Health Center (Epicentro) in Lima, Peru, HIV prevalence in 2010 was approximately 8\% (unpublished data). Along with the elevated HIV prevalence in MSM, the prevalence of HPV in MSM in any anatomical site in the US approaches $50 \%{ }^{10}$ but data on HPV in MSM in Peru are unknown.

Nonetheless, anecdotal evidence from STI clinics in Peru suggests a high burden of the clinical manifestation of HPV, that is, AGW, in the anal, perianal and penile regions. At Epicentro, for example, AGW is the major symptom of patients presenting for care and accounts for over $35 \%$ of all medical consults received at the centre (clinic data, personal communication). While multiple HIV prevention investigations are underway (behavioural and clinical) in Peru, none has reported the prevalence of AGW in MSM/TGW nor considered their role in HIV acquisition. These data are crucial since anal condyloma is a significant risk factor for anal squamous intraepithelial lesions in HIV positive MSM. ${ }^{20}$

This novel study proposes to measure the prevalence of AGW in MSM/TGW presenting at a community clinic environment and prospectively measure HIV incidence in those with AGW and those without AGW. It will be the first study that we are aware of using HIV infection as an end point in MSM/TGW with and without AGW and will help to better understand the relationship between AGW and HIV infection among these populations in Peru.

\section{METHODS AND ANALYSIS}

Overview: the proposed protocol was developed and facilitated by collaborations between experienced senior investigators from UPCH, University of California, Los Angeles (UCLA), University of California, Irvine (UCI) and Epicentro. The study will follow a cohort of 600 MSM/TGW, half with and half without AGW to determine the role of AGW in HIV acquisition. A sample size of $264 \mathrm{MSM} / \mathrm{TGW}$ in each group (one group with AGW and one without) will provide $80 \%$ power to detect an $8 \%$ difference in HIV incidence in the groups. Studying the role of AGW in HIV acquisition is important in this group because of the common way both STIs are transmitted and the high prevalence of HIV among MSM/ TGW in Peru.

\section{Objectives}

The primary objective of this study is to determine the role of AGW on HIV acquisition among MSM/TGW in Peru. The secondary objectives are to determine HPV prevalence in HIV positive MSM/TGW in Peru, risk factors associated with AGW, and the knowledge of HPV and HIV among MSM/TGW. The specific aims of this study are to (1) estimate HIV incidence in Peruvian MSM/TGW by AGW status, (2) to determine the prevalence of HIV among Peruvian MSM/TGW by AGW status, (3) to determine the type-specific prevalence of anal HPV infection in HIV positive Peruvian MSM/TGW.

\section{Study design/population}

This prospective clinical study will consist of surveys and specimen collection from a cohort of $600 \mathrm{MSM} / \mathrm{TGW}$. At baseline we will examine participants for AGW presence and collect information on the history of AGW. We will select $300 \mathrm{MSM} / \mathrm{TGW}$ with current AGW and 300 without AGW for the study, then follow-up with HIV and HPV testing every 6 months for 2 years. A survey will be administered at each visit that examines changes in risk behaviours over time, by HIV status and by AGW status. Participants will undergo a physical examination to look for current STI, including AGW. Participants will also be screened for HPV genotypes, syphilis and herpes at baseline (figure 1).

\section{Inclusion criteria}

MSM and TGW will be invited to join the study if they meet the following criteria (1) anatomical men 1840 years of age, (2) self-reported anal sex with a man within 12 months prior to enrolment, (3) willing to provide informed consent for the collection of demographic and sexual behaviour data, as well as blood for HIV and syphilis testing, swabs of anal mucosa for HPV testing and anal swabs for chlamydia testing, and (4) resident of metropolitan Lima. Additional criteria apply for the study arm with AGW, in that participants must have either current AGW or a prior episode of AGW. Participants will be excluded if they are HIV positive, had prior participation in an HIV or HPV clinical trial, if they have a known immunodeficiency that increases the risk of acquiring HIV, or if they self-report use of preexposure prophylaxis (PrEP). 


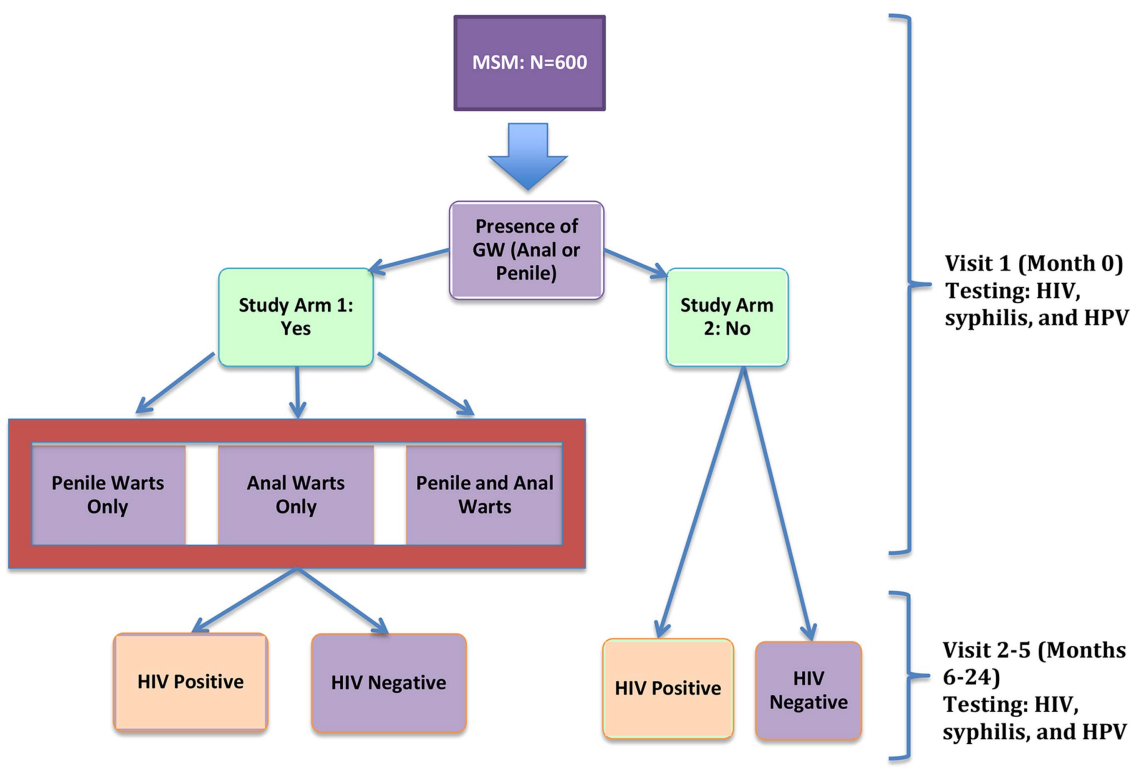

Figure 1 Flowchart of participant enrolment and study procedures for $600 \mathrm{MSM}$.

\section{Study procedures}

Recruitment

Participants will be recruited at their regular visits to Epicentro, and outreach activities specified below. We will recruit the first $300 \mathrm{MSM} / \mathrm{TGW}$ in each group (with and without AGW) who agree to participate and meet eligibility criteria, and we expect to enrol 5-20\% TGW based on current visitors at Epicentro (unpublished data). Should our eligible population be exhausted at these locations since there are no published studies or data on recruiting participants with AGW in Peru, we will undertake additional recruitment procedures including fieldwork, utilising social media and snowball sampling (table 1).

\section{Physical examination}

At each study visit, all participants will have a simple clinical examination to check for signs of current AGW. If medical diagnosis of AGW is uncertain, or the lesion is atypical, confirmation will be done with a biopsy. All participants will be informed about AGW, shown detailed pictures, and discuss doubts with medical personnel to better understand if they have had an outbreak in the past. Participants who report having an outbreak of AGW in the past 6 months, or who are shown to have AGW during the clinical examination will be placed in the AGW group. AGW types will be stratified into anal only, penile only and both anal and penile. Incident AGW cases in the non-AGW group will be counted, with incident AGW treated as a study outcome. Non-identifiable photos of the AGW will be taken and shared with study clinicians to confirm presence. Participants with anal lesions and abnormalities will be referred to a specialist and will receive anoscopy, while AGW will be treated with podophyllin or liquid nitrogen (table 2). Syndromic management will be followed per Peruvian national guidelines for other STIs. ${ }^{21}$

\section{Behavioural survey}

A computer assisted self-interview behavioural survey will be administered at each study visit over the 2-year period. We will collect information on the awareness of

Table 1 Recruitment plans for 600 study participants with and without AGW

\begin{tabular}{ll}
\hline Item & Description \\
\hline Field work & Peer educators will visit socialisation locations of MSM/TGW to distribute information materials about \\
& the project and invite them to participate \\
& Team will consist of MSM/TGW with different sexual self-identities (gay, heterosexual, bisexual, \\
& transgender) to ensure sample heterogeneity \\
Social media & Dissemination of the project through social networks (Facebook, Twister and hi5) \\
& Contact potential participants via chat rooms, gay ads in the most popular Peruvian webpage's \\
(gayperu, peruesgay), and diffusion through blogs related to sexual health \\
sampling & In counselling sessions, enrolled participants asked to invite their friends, partners and acquaintances \\
& to participate
\end{tabular}


Table 2 Genital wart treatment options for study participants

\begin{tabular}{llllll}
\hline Treatment & Frequency & $\begin{array}{l}\text { Number of } \\
\text { sessions }\end{array}$ & Area & Where & Treatment time \\
\hline Podofilin (25\%) & Weekly & $2-5$ & $2 \mathrm{~cm}$ diameter & Skin & N/A \\
Cryotherapy & $10-15$ days & $2-8$ & $2 \mathrm{~cm}$ diameter & $\begin{array}{l}\text { Skin, mucous } \\
\text { membrane }\end{array}$ & $\begin{array}{l}5-20 \mathrm{~s} \text { with } 10-40 \mathrm{~s} \\
\text { between applications }\end{array}$ \\
\hline N/A, not applicable & & & & &
\end{tabular}

AGW and its relationship with HIV, risk factors for HIV incidence, knowledge of HPV and HPV vaccine, penile washing, STI history, age, condom use, number of sexual partners, smoking, drug/alcohol use, sexual role (insertive or receptive partner) and current STIs. Utilising the same survey questions at each visit will allow us to view changes in behaviours by AGW and HIV status over time. Encrypted SurveyMonkey will be used for data collection. No personal identifiers will be collected, and the survey will take place in a private room by the participant. At the baseline visit, the survey will be carried out following the physical examination to ensure we receive 300 participants with AGW. In subsequent visits, the survey will be carried out prior to the physical examination and STI testing.

\section{HIV testing and counselling}

We will perform HIV rapid testing using determine HIV-1/2 Ag/Ab Combo rapid test. Before conducting the rapid HIV test, a trained counselor will provide HIV/STI counselling. Results will be disclosed during a post-test counselling session during the same visit. Participants will be asked to provide a venous blood specimen for the standard immunoassay test ELISA for confirmation of a positive rapid test result. If positive by ELISA, the participant will be linked to the Peruvian National HIV Treatment Programme (Programa Targa) for assessment of highly active antiretroviral therapy (HAART) treatment. Rapid and ELISA testing will take place at each of the 5 study visits across the 2-year study for men who are HIV negative.

\section{HPV sampling and testing}

Anal samples for the detection of HPV will be collected by trained medical staff using Digene sampler kits at each study visit. First, the study clinician will visually examine the anus for noticeable lesions. Next, the clinician will examine the anus and insert the small wet swab approximately 1-2 inches into the anal cavity, and store the swab for HPV testing. Two additional wet swabs will be taken, one of the penile shaft and one of the scrotum. Samples will be analysed for the presence or absence of HPV DNA by PCR using PGMY09/PGMY11 primers at Johns Hopkins Bloomberg School of Public Health.12 Roche Linear Array genotyping testing detected 37 HPV types including carcinogenic $(16,18$, $31,33,35,39,45,51,52,56,58,59,66)$ and noncarcinogenic types $(6,11,26,40,42,53,54,61,62,64$,
$67,68,69,70,72,73,81,82,82$ var, 83, 84 and 89) as defined at the 2005 meeting of the International Agency for Research on Cancer. Only $\beta$-globin positive samples will be included in testing.

\section{Blood draw for HPV antibodies}

At baseline and on the final visit, we will collect $6 \mathrm{~mL}$ of blood to test for HPV4 antibodies in order to characterise the history of HPV infection and compare with HPV DNA prevalence. These data provide information on previous HPV infection not necessarily detectable by HPV DNA testing, as shown in previous studies. ${ }^{22}$

\section{STI testing and counseling}

All participants will undergo a short session of counselling on how to avoid STIs and on correct condom use at each study visit. Condoms and lubricants will be provided at each visit. At baseline, all participants will be tested for syphilis with a rapid test by means of a finger prick. All participants who test positive will receive a single intramuscular injection of Penicillin according to Peruvian national guidelines. Medical staff will obtain an anal swab sample from participants at visits 2-5 which will be tested for chlamydia and gonorrhoea. The treatments for syphilis and other STIs will be administered by licensed clinicians and will follow the Peruvian national guidelines for their treatment (table 3) ${ }^{21}$

\section{Incentives and retention}

Study participants will receive a drink and snack at the initial visit which is expected to last approximately $2 \mathrm{~h}$ with the physical examination and survey. They will also receive a small gift at each visit (approximate value USD $\$ 4)$ as well as condoms and lubricants. Additionally, participants will receive monthly calls and emails between appointments if they do not come to the Epicentro clinic in the interim. Participants who do not return for visits will be followed up at home and work.

\section{Statistical methods and analysis}

Personal identifiers will be unlinked prior to analysis. No blinding will occur since this is not a randomised controlled trial and all study procedures are the same for each participant. Numerical interview data will be exported into EpiInfo and checked for entry errors, logic and range. We will use normal $\chi^{2}$ test for univariate analyses of binary variables, and Fisher's exact test when the sample is small. Student's t test and Mann-Whitney 


\begin{tabular}{|c|c|}
\hline $\begin{array}{l}\text { Etiological } \\
\text { agent }\end{array}$ & Treatment \\
\hline Syphilis & $\begin{array}{l}\text { Benzathine G penicillin } 2.4 \mathrm{MU} \text { in two } \\
1.2 \mathrm{MU} \text { intramuscular injections once. } \\
\text { If allergy to penicillin exists: } \\
\text { Doxiciclyne } 100 \mathrm{mg} \text { orally. Twice daily } \\
\text { per } 14 \text { days }\end{array}$ \\
\hline Gonorrhoea & $\begin{array}{l}\text { Cefixime } 400 \mathrm{mg} \text { orally once. } \\
\text { Or } \\
\text { Ceftriaxone } 125 \mathrm{mg} \text { intramuscular once. }\end{array}$ \\
\hline Chlamydia & $\begin{array}{l}\text { Azithromicin } 1 \mathrm{~g} \text { orally once or } \\
\text { Doxiciclyne } 100 \mathrm{mg} \text { orally each } \\
12 \mathrm{~h} \times 7 \text { days }\end{array}$ \\
\hline Trichomonas & Metronidazole $2 \mathrm{~g}$ orally once \\
\hline
\end{tabular}

$\mathrm{U}$ test will be used for analysis of continuous variables. Multivariate analyses will be performed using HRs to assess variables associated with HIV incidence in participants with AGW and without. The incidence and prevalence of HIV will be measured for AGW overall and separately for the different types (penile only, anal only and both). We will present prevalences and 95\% CIs for HPV types both DNA and serology. Analyses will be performed using Stata (V.10.0; Stata Corp., College Station, Texas, USA). Numerical interview data will be double entered into EpiInfo and checked for logic and range.

\section{Power/sample size}

To determine the sample size for our primary objective, we used Stata V.10.0. A sample size of $264 \mathrm{MSM} / \mathrm{TGW}$ in each group will provide $80 \%$ power to detect an $8 \%$ difference in HIV incidence over 2 years $(15 \%$ among those with AGW, and 7\% among those without AGW; HR: 2.1; with a type I error of 0.05 . We have an expected $13 \%$ of loss of follow-up; therefore, we will need 299 participants in each group.

\section{ETHICS AND DISSEMINATION}

The study protocol has been approved by the UCLA and Impacta Institutional Review Boards with a start date of 15 February 2012 and end date of 27 September 2015. Epicentro has been involved in several studies including the IISP study of HPV in MSM/TGW, the acceptability of rectal microbicides, HIV sentinel surveillance and a study of HIV prevention in male sex workers in Lima, Peru. We anticipate that the benefits of study participation will outweigh any risks, including loss of confidentiality and privacy of clinical information. Participants will benefit from treatment of newly-diagnosed STIs, treatment of genital warts, linkage to treatment for HIV infection when indicated, and optional activities in becoming part of the only gay men's and transgender women's community health centre in Peru. Results from this study may facilitate the development of proactive intervention strategies to reduce the prevalence of AGW and hence prevent incident HIV infection.
Among MSM/TGW, HPV infection has been reported to be associated with specific sexual practices, such as receptive anal intercourse, which in turn is associated with increased risk of acquiring HIV. It is therefore realistic to believe that MSM/TGW who are HPV-infected may have been at risk for exposure to HIV infection because both viruses are predominately contracted through common sexual practices. The proposed study will attempt to measure the prevalence of AGW and the incidence of HIV among participants with and without AGW over a 36-month period. This novel and adequately powered approach may help us better understand the relationship between HPV-related AGW and HIV acquisition in MSM/TGW in Peru and how to reduce the prevalence of both. While there have been several studies indicating a possible relationship between HPV and HIV, studies that illustrate a temporal association between HPV-related AGW and HIV acquisition are significantly lacking. STI co-infection including HPV-related manifestations may be a good proxy for HIV risk behaviours.

Knowledge gained from this study may be used to design similar protocols with a higher number of participants and appropriate intervention strategies to reduce the burden of HPV-related diseases and HIV among a particularly vulnerable population group who is heavily burdened by both. Additionally, to make certain that national and international scientific communities can access our study findings and results, we will share them within a multitude of forums including the Peruvian Ministry of Health as well as other public health organisations that can further assist with the dissemination and institutionalisation of pertinent information.

\section{Author affiliations}

${ }^{1}$ Program in Public Health, Department of Population Health and Disease Prevention, University of California, Irvine, California, USA

${ }^{2}$ Unit of Health, Sexuality and Human Development, Cayetano Heredia University School of Public Health, Lima, Peru

${ }^{3}$ Epicentro Salud, Lima, Peru

${ }^{4}$ Division of Infectious Diseases, Department of Medicine, University of California, Los Angeles, USA

Acknowledgements The authors thank the UCLA Program in Global Health and Investigators at the Universidad Peruana Cayetano Heredia School of Public Health for their initial review and feedback on the study proposal. The authors would like to thank the staff at Epicentro for their assistance in the study procedures and operations.

Contributors BB and JG are the principal investigators who conceived the study design, and supervised the study, and provided critical revision of the manuscript. MD helped draft the first version of the manuscript. SRL assisted with the manuscript, created the laboratory manual of procedures, and assisted with testing of study samples. HS and GC provided feedback on the study design, created the protocols for participant recruitment and retention, and helped coordinate study visits. JDK is a senior investigator who provided leadership on the study and manuscript, and assisted with additional study procedures. All authors read and approved the final manuscript.

Funding Supported in part by a research grant from the Merck Investigator-Initiated Studies Program of Merck \& Co., Inc (grant number 39619).

Competing interests None. 
Ethics approval UCLA IRB and Impacta ethics committee.

Provenance and peer review Not commissioned; externally peer reviewed.

Data sharing statement This is a study protocol submission.

Open Access This is an Open Access article distributed in accordance with the Creative Commons Attribution Non Commercial (CC BY-NC 4.0) license, which permits others to distribute, remix, adapt, build upon this work noncommercially, and license their derivative works on different terms, provided the original work is properly cited and the use is non-commercial. See: http:// creativecommons.org/licenses/by-nc/4.0/

\section{REFERENCES}

1. Brown B, Davtyan M, Galea J, et al. The role of human papillomavirus acquisition in men who have sex with men: a review of the literature. Viruses 2012;4:3851-8.

2. Smith JS, Moses S, Hudgens MG, et al. Increased risk of HIV acquisition among Kenyan men with human papillomavirus infection $J$ Infect Dis 2010;201:1677-85.

3. Rottingen JA, Cameron DW, Garnett GP. A systematic review of the epidemiologic interactions between classic sexually transmitted diseases and HIV: how much really is known? Sex Transm Dis 2001;28:579-97.

4. Kuiken CL, Van Griensven GJ, De Vroome EM, et al. Risk factors and changes in sexual behavior in male homosexuals who seroconverted for huOman immunodeficiency virus antibodies. Am J Epidemiol 1990;132:523-30.

5. Laga M, Manoka A, Kivuvu M, et al. Non-ulcerative sexually transmitted diseases as risk factors for HIV-1 transmission in women: results from a cohort study. AIDS 1993;7:95-102.

6. Cameron DW, Simonsen JN, D'costa LJ, et al. Female to male transmission of human immunodeficiency virus type 1: risk factors for seroconversion in men. Lancet 1989;2:403-7.

7. Craib KJ, Meddings DR, Strathdee SA, et al. Rectal gonorrhoea as an independent risk factor for HIV infection in a cohort of homosexual men. Genitourin Med 1995;71:150-4.

8. Freeman EE, Weiss HA, Glynn JR, et al. Herpes simplex virus 2 infection increases HIV acquisition in men and women: systematic review and meta-analysis of longitudinal studies. AIDS 2006;20:73-83.

9. Jin F, Prestage GP, Imrie J, et al. Anal sexually transmitted infections and risk of HIV infection in homosexual men. J Acquir Immune Defic Syndr 2010;3:144-9.

10. Goldstone S, Palefsky JM, Giuliano AR, et al. Prevalence of and risk factors for human papillomavirus (HPV) Infection among HIV-seronegative men who have sex with men. J Infect Dis 2011;203:66-74.

11. Machalek DA, Poynten M, Jin F, et al. Anal human papillomavirus infection and associated neoplastic lesions in men who have sex with men: a systematic review and meta-analysis. Lancet Oncol 2012b;13:487-500.

12. Donà MG, Palamara G, Di Carlo $A$, et al. Prevalence, genotype diversity and determinants of anal HPV infection in HIV-uninfected men having sex with men. J Clin Virol 2012;54:185-9.

13. Chin-Hong PV, Husnik M, Cranston RD, et al. Anal human papillomavirus infection is associated with HIV acquisition in men who have sex with men. AIDS 2009;23:1135-42.

14. MSM, HIV, and the Road to Universal Access. How Far Have We Come? New York: The Foundation for AIDS Research, 2008.

15. UNAIDS. Report on the global AIDS epidemic: 2008.

16. Caceres CF, Mendoza W. Monitoring trends in sexual behaviour and HIV/STIs in Peru: are available data sufficient? Sex Transm Infect 2004;80(Suppl 2):ii80-4.

17. Bautista CT, Sanchez JL, Montano SM, et al. Seroprevalence of and risk factors for HIV-1 infection among South American men who have sex with men. Sex Transm Infect 2004;80:498-504.

18. UNAIDS. Global report: UNAIDS report on the global AIDS epidemic 2010. 2010:364.

19. Clark JL, Segura ER, Montano SM, et al. Routine laboratory screening for acute and recent HIV infection in Lima, Peru. Sex Transm Infect 2010;86:545-7.

20. Li AH, Phanuphak N, Sahasrabuddhe VV, et al. Anal squamous intraepithelial lesions among HIV positive and HIV negative men who have sex with men in Thailand. Sex Transm Infect 2009;85:503-7.

21. MINSA. Norma Técnica de salud para el manejo de infecciones de transmisión sexual en el Perú. 2009

22. Brown B, Blas M, Cabral A, et al. Randomized trial of HPV4 vaccine assessing the response to vaccine in two schedules among Peruvian FSWs. Vaccine 2012;30:2309-14. 